\title{
Revitalizing monoamine oxidase inhibitors: a call for action
}

\author{
P. Ken Gillman, ${ }^{1 *}$ S. Shalom Feinberg, ${ }^{2}$ and Laura J. Fochtmann ${ }^{3}$ (1)
}

\author{
${ }^{1}$ PsychoTropical Research, Queensland, Australia \\ ${ }^{2}$ Department of Psychiatry and Behavioral Science, Albert Einstein College of Medicine, Montefiore Medical Center, Bronx, NY, USA \\ ${ }^{3}$ Departments of Psychiatry, Pharmacological Sciences and Biomedical Informatics, Stony Brook University, Stony Brook, NY, USA
}

Received 16 May 2019; Accepted 24 May 2019

This statement represents the view of the "International MAOI Expert Group" which is a group of clinicians, academics, and researchers with a special interest in monoamine oxidase inhibitors (MAOIs). This group was formed in March 2018 to promote actions aimed at: improving education; stimulating research; increasing clinical usage; and assuring continued availability of MAOIs worldwide.

Non-selective irreversible MAOIs, including tranylcypromine, phenelzine, isocarboxazid, and the Selegiline Transdermal System (STS), can be dramatically effective for patients with biological depressive illnesses (e.g., melancholia and bipolar depression). It has been established by more than 50 years of research, including randomized double-blind studies, and clinical experience, that these medications can induce full remission when other antidepressants, combinations, and augmentation strategies, and even electroconvulsive therapy (ECT), have failed. ${ }^{1-4}$ Because of their unique pharmacological properties, the efficacy of non-selective irreversible MAOIs is superior, in a proportion of patients, to more recently developed medications, including moclobemide, a reversible inhibitor of MAO-A, or MAO-B-selective doses of selegiline. Patients may experience fewer side effects with MAOIs than with other antidepressants; for example, the low rates of weight gain, cognitive dysfunction and sexual side effects with tranylcypromine and selegiline (STS) are a major advantage for many patients. ${ }^{5,6}$ Despite these valuable attributes these vital life-saving antidepressant drugs are becoming unaffordable (because the cost has inexplicably escalated by about 50-fold), less available, or even unavailable, in many countries around the world.

* Address correspondence to: Ken Gillman, PsychoTropical Research, PO Box 86, Rural View, Queensland 4740, Australia. (Email: ken.psychotropical@gmail.com)
Usage of MAOIs began to decline several decades ago following the development of newer antidepressants that were initially supposed to have greater efficacy, fewer side effects, and lower rates of mortality in overdose: those suppositions were unfulfilled, or only partially fulfilled. As more newer antidepressants became available the number of individuals treated with MAOIs has steadily decreased; thus, many patients have been denied an opportunity for effective treatment. Another reason may be that an over-emphasis on possible risks of MAOI treatment has overshadowed full recognition of the impact of the distress and morbidity of undertreated and untreated depression, which may be underestimated or forgotten. Clinical experience and research show that many individuals suffering with the heterogenous entity labeled as "depression", including those with severe melancholia-type illnesses and bipolar depression, do not respond well to the newer antidepressants, nor to combination or augmentation strategies, but may respond to MAOIs. ${ }^{7}$ The enormous cost to society and the health-care system of partially and ineffectively treated depressive illness is well documented and results in depression-related disability and suicide. MAOIs can save society, health-care insurers, and providers considerable costs. Even more importantly from a clinical-care point of view, the under-usage of MAOIs has caused much prolonged and needless suffering and avoidable deaths. Thus, it is crucial to have these essential drugs available in the clinician's armamentarium and to have clinicians who are knowledgeable about their usage.

It is rare for psychiatrists in training to be educated about MAOIs, so they graduate from otherwise excellent training programs without any knowledge of MAOI treatment and not knowing that MAOIs can be used safely and effectively to improve outcomes for patients.

Education tends to focus on recent literature and rigorous systematic reviews; we contend there is insufficient 
emphasis on the rich early data on MAOIs as well as decades of successful clinical experience with their usage. Consequently, psychiatrists overestimate the risks of side effects, drug-drug interactions, and the difficulty of following a low-tyramine diet with MAOIs. ${ }^{8,9}$ It has been pointed out that there is much misleading and incorrect information contained in standard texts, including the United States Physicians' Desk Reference (PDR), the European electronic Medicines Compendium (eMC), and the British National Formulary (BNF); these texts require urgent updating to remove errors and bring them into line with state-of-the-art knowledge. Texts with more up-todate information are available. , $^{8,10}$

These issues lead to a disproportionate "riskaversion" toward prescribing MAOIs. Other examples of commonly perpetuated but incorrect information include the ideas that changes to medication regimens or combinations of antidepressants are difficult when receiving an MAOI, that MAOIs must be stopped prior to anesthesia, and that opioid medications cannot be used., ${ }^{5,9}$ Newer medications also have risks, albeit different ones, but experience with them during training leads to greater comfort in prescribing them, as compared with MAOIs.

It is suggested that professional organizations, healthcare teaching institutions, and regulatory bodies should facilitate improvements concerning MAOI-related issues, and that specialists should become proficient in MAOI usage. In addition to strengthening psychiatric training related to MAOIs, several other approaches can be taken to achieve these goals. Audits of practice and performance improvement programs have been developed for other treatments (e.g., clozapine, lithium, ECT) to monitor adherence to best practices and provide feedback to assist in developing and maintaining knowledge and skills. Continuing education programs should also be developed to help physicians review, update, and test their knowledge about MAOIs.

Given the increasing emphasis on evidence-based medicine (EBM), it is also essential to emphasize and adhere to EBM tenets: these require "integrating ... the best available external clinical evidence from systematic research ... [with] the proficiency and judgment that individual clinicians acquire through clinical experience and clinical practice... [without which] even excellent external evidence may be inapplicable to or inappropriate for an individual patient." 11 Despite Sackett's injunction, the importance of patient experience and preferences, and clinical expertise, are commonly underemphasized in the care delivered to individuals, and in the synthesizing of evidence for inclusion in clinical practice guidelines. ${ }^{12}$ Practice guideline developers might give greater consideration to incorporating more information about MAOIs from clinical expertise and patient perspectives, in addition to the limited evidence-based information from trials (which may be of uncertain generalizability).

Enhanced attention to MAOIs in clinical practice guidelines will help promote best practices on MAOI usage and also improve the education of current and future physicians. Improvements in education and clinical practice guidelines, in combination with advocacy efforts to assure continued availability of MAOIs worldwide, will help patients continue to reap the benefits of these life-saving medications.

\section{Summary: Expert group recommendations}

We recommend:

1. That bodies such as professional organizations, healthcare teaching institutions, and regulatory agencies act to facilitate the availability in their countries of all four MAOI medications (i.e., tranylcypromine, phenelzine, isocarboxazid, and STS).

2. That pharmacy databases, drug-drug interaction software, electronic health records, and patient educational materials require urgent updating to incorporate correct state-of-the-art information on drug interactions and on dietary advice.

3. That specialty training in psychiatry should incorporate knowledge of, and experience in, the clinical use of all MAOIs.

4. That practice guideline developers incorporate information about MAOIs from older literature, clinical expertise, and patient perspectives to provide a more complete view of the important role of MAOIs in clinical practice.

5. That there be improvement of continuing education about MAOIs and performance initiatives to audit MAOI prescribing, provide feedback to prescribers, and assure use of best practices with MAOIs.

\section{Additional signatories}

Additional signatories to this statement are: Martin Alda, Ian Anderson, Michael Bauer, David Baldwin, Franck J Baylé, Michael Berk, Tom Birkenhager, Marc Blom, J. Alexander Bodkin, Robert Bransfield, Carlo Ignazio Cattaneo, Alastair Clarke-Walker, Anthony Cleare, William Coryell, John Cosgrove, David Cousins, Phil Cowen, Jonathan Davidson, Jackson Dempsey, J. Raymond DePaulo Jr., Michel Dierick, Michael Feinberg, Carlos Fernandez, Angelo Ferrero, Jess G. Fiedorowicz, Eric Fier, John Finberg, James Flax, Michele Fornaro, Joanna Fowler, Michael J. Gitlin, Luis Giuffra, Guy Goodwin, John Greden, Peter Haddad, Shmuel Harris, Nicolas Hoeh, Andrew Holt, Harish Kavirajan, Sidney Kennedy, Donald Klein, Michael Kornberg, Jens Knud Larsen, Gerd Laux, 
William McDonald, Hamish McAllister-Williams, David Menkes, Thomas Messer, Jonathan Meyer, Paul Miller, Michael Miller, Darrell Mousseau, Willem Nolen, David Nutt, Sagar V. Parikh, Gordon Parker, Frenk Peeters, Rona Ramsay, Michelle Riba, Elliott Richelson, Mark Rowe, Robert Rubin, Jeronimo SaizRuiz, Max Schmauss, Robert Schoevers, Stuart Seidman, Sudhakar Selvaraj, Jean Chen Shih, Balwinder Singh, Jair C Soares, Stephen Stahl, Clare Stanford, Thomas Steele, Jeffrey R. Stenzel, Peter Talbot, Davin Tan, David Tobolowsky, Sven Ulrich, Ian Whyte, Daniel Winstead, Jesse H. Wright, and Allan Young.

\section{Acknowledgments}

The authors wish to acknowledge Heidi Counsell, Senior Information Technology and Administrative Assistant for her invaluable assistance in the preparation of this manuscript.

\section{Disclosures}

Dr. Gillman has a patent 62/806.831 pending for an altered formulation of tranylcypromine. In addition, he has equity interests in NeuraWell, which is a company that is obtaining the patent and has been formed in an attempt to remarket tranylcypromine. Dr. Feinberg has nothing to disclose. Dr. Fochtmann reports that she consults for the American Psychiatric Association on the development of practice guidelines, that her institution receives salary offset for these services and she has been compensated for travel to attend meetings related to these duties.

\section{REFERENCES:}

1. Ricken R, Ulrich S, Schlattmann P, et al. Tranylcypromine in mind (part II): review of clinical pharmacology and meta-analysis of controlled studies in depression. Eur Neuropsychopharmacol. 2017; 27(8): 714-731.

2. Amsterdam JD, Shults J. MAOI efficacy and safety in advanced stage treatment-resistant depression-a retrospective study. J Affect Disord. 2005; 89(1-3): 183-188.

3. Shulman KI, Fischer HD, Herrmann N, et al. Current prescription patterns and safety profile of irreversible monoamine oxidase inhibitors: a population-based cohort study of older adults. J Clin Psychiatry. 2009; 70(12): 1681-1686.

4. Birkenhager TK, van den Broek WW, Mulder PG, et al. Efficacy and tolerability of tranylcypromine versus phenelzine: a double-blind study in antidepressant-refractory depressed inpatients. J Clin Psychiatry. 2004; 65(11): 1505-1510.

5. Adli M, Pilhatsch M, Bauer M, et al. Safety of high-intensity treatment with the irreversible monoamine oxidase inhibitor tranylcypromine in patients with treatment-resistant depression. Pharmacopsychiatry. 2008; 41(6): 252-257.

6. Razani J, White KL, White J, et al. The safety and efficacy of combined amitriptyline and tranylcypromine antidepressant treatment. A controlled trial. Arch Gen Psychiatry. 1983; 40(6): 657-661.

7. Shulman KI, Herrmann N, Walker SE. Current place of monoamine oxidase inhibitors in the treatment of depression. CNS Drugs. 2013; 27(10): 789-797.

8. Gillman PK. A reassesment of the safety profile of monoamine oxidase inhibitors: elucidating tired old tyramine myths. J Neural Transm (Vienna). 2018; 125(11): 1707-1717.

9. Gillman PK. "Much ado about nothing": monoamine oxidase inhibitors, drug interactions, and dietary tyramine. CNS Spectr. 2017; 22(5): 390 .

10. Stahl SM. Stahl's Essential Psychopharmacology: The Prescribers Guide. 6th ed. New York, NY: Cambridge University Press; 2017.

11. Sackett DL, Rosenberg WM, Gray JA, et al. Evidence based medicine: What it is and what it isn't. BMJ. 1996; 312(7023): 71-72.

12. Greenhalgh T, Snow R, Ryan S, et al. Six 'biases' against patients and carers in evidence-based medicine. BMC Medicine. 2015; 13: 200. 